\title{
Laser cavity mirror imperfections and reflectivity: A time-dependent numerical approach
}

\author{
Igor Vurgaftman and Jasprit Singh \\ Solid State Electronics Laboratory, Department of Electrical Engineering and Computer Science, \\ The University of Michigan, Ann Arbor, Michigan 48109-2122
}

(Received 22 August 1994; accepted for publication 9 November 1994)

\begin{abstract}
While the cleaving process used for semiconductor Fabry-Pérot lasers produces atomically abrupt mirrors, there is considerable interest in mirrors defined by etching. Depending on the etching process employed, disorder of varying nature and degree results at the semiconductor-air interface. A theoretical approach capable of quantifying the impact of such disorder on the mirror reflectivity, to which laser performance is intimately connected, is presented. The theoretical technique is time-dependent to facilitate visualization of the scattering process and is based on a locally one-dimensional implicit-finite-difference approximation to the two-dimensional scalar wave equation with variable coefficients. Mirror disorder is described in terms of a feature depth parameter and an in-plane correlation length. The reflectivity falls off exponentially with disorder yet is found to remain close to its unperturbed value for the disorder scale attainable with the state-of-the-art etching technology. (C) 1995 American Institute of Physics.
\end{abstract}

The accepted technique for mirror definition in semiconductor laser cavities is the cleaving of the substrate. This approach has the advantage of producing vertical and atomically flat facets, yet at the same time dictates the discretedevice nature of the laser fabrication technology and makes the integration of lasers with optical and electronic components difficult, if not impossible. Therefore, in recent years, there have been numerous attempts to fabricate laser mirrors by various etching techniques. ${ }^{1-4}$ Experimental studies and simple calculations show that with high-quality etching procedures there is minimal degradation of the mirror quality produced by etching. However, it is not a priori clear how a particular etching process can lead to positive results in laser fabrication since the mechanism of optical wave reflection and scattering from a disordered interface cannot be modeled analytically with any degree of confidence owing to its complicated details. Moreover, since the roughness pattern left by the etching process is not reproducible, it is important to have a quantitative understanding of the extent of variability in the reflectivity and, consequently, laser performance for successive applications of the same etching technique. In this letter, we present a straightforward and efficient numerical model allowing accurate estimates of the reflectivity and scattering losses from a homogeneously disordered interface placed at the end of a slab waveguide. This model permits us to understand quantitatively and visualize the processes leading to the reduction of the mirror reflectivity as a function of the degree of their roughness. The model can also be applied to more general cases of wave packet propagation in optical devices and components, such as directional couplers, fiberto-fiber couplings etc., wherein imperfections and spatial disorder may reside.

Our model is based on a two-dimensional timedependent scalar wave equation with variable refractive index specialized to TE-polarized waveguide modes. If $E_{x}(y, z, t)$ can be expressed as a linear combination of plane waves of the form $\exp [i(\beta z-\omega t)]$ by assuming translational invariance in the $z$-direction, the wave equation can be separated in the longitudinal $(z)$ and transverse $(y)$ coordinates $\left[E_{x}(z, y, t)=\epsilon_{x}(z, t) \phi(y)\right] . \beta$ is the longitudinal wave number, and $\omega$ is the angular frequency eigenvalue. The solution of the $1 \mathrm{D}$ wave equation in the general case is well known, ${ }^{5}$ and has been obtained in this work by the finite-difference approximation to the second derivative term.

To formulate the starting condition for the timedependent wave propagation problem, a $2 \mathrm{D}$ wave packet is constructed from a continuum of forward-propagating plane waves centered around some wavelength $\lambda_{0}$ (here $\left.\lambda_{0}=\lambda_{\text {vacuum }} / n_{\text {mat }}\right)$ and the stationary fundamental-mode transverse profile

$$
\begin{aligned}
E_{x}(y, z, t)= & \operatorname{Re}\left[\phi_{0}(y) \int \exp \left(-\frac{\left(\beta-\beta_{0}\right)^{2}}{2(\Delta \beta)^{2}}\right)\right. \\
& \left.\times e^{i\left[\beta\left(z-z_{0}\right)-\omega t\right]} d \beta\right],
\end{aligned}
$$

where $\Delta \beta$ is chosen to be a small fraction of $2 \pi / \lambda_{0}$. The wave packet (1) is a solution of the wave equation in the waveguide with a uniform refractive index profile along the longitudinal axis. Since such a wave packet maintains its shape propagating in the waveguide at some group velocity $c / n_{\text {eff }}$, it is ideally suited for studying wave reflection, transmission, and scattering from interfaces placed at the end of a uniform waveguide region $z_{i}$. These interfaces do not interfere with the initial wave packet construction provided $\left|z_{0}-z_{i}\right| \gg(\Delta \beta)^{-1}$. To insure stability and precision of the numerical technique ${ }^{6}$ we resort by resorting to an implicit difference approximation. It is convenient to choose a locally one-dimensional scheme ${ }^{7}$ and generalize it to the case of variable coefficients $n_{l, m}$. This approximation scheme is unconditionally stable and has a principal truncation error of 
$O\left(\Delta y^{3}+\Delta t^{3}\right)$. The details of the derivation rooted in a sequence of algebraic manipulations will be presented in a separate, lengthier, report.

Noting that finding the function value at the most advanced time step involves, at most, inverting a tridiagonal matrix and invoking the boundary conditions $U_{0, m}^{n}=U_{l_{\max }, m}^{n}=U_{l, 0}^{n}=U_{l, m_{\max }}^{n}=0$, where $U$ is the weight amplitude for all $l, m, n$, in the $y$ and $z$ directions and time, respectively, we select the Thomas algorithm ${ }^{7}$ as an efficient method of solving the matrix equation. In order to be able to apply the Thomas algorithm, it is necessary that for the worst-case situation $n_{l, m}=n_{l-1, m}=n_{1}$ and $n_{l+1, m}=n_{2}$ (the indices $l, m$ are, of course, interchangeable in this context), the condition

$$
\frac{4 n_{1} n_{2}}{p^{2}} \geqslant \frac{n_{1}-n_{2}}{n_{1}},
$$

where $p=c \Delta t / \Delta y$, and $\Delta y=\Delta z$, be satisfied. Taking $n_{2}$ to have its lowest possible value 1.0 , and choosing $p=n_{1}$ for convenience in monitoring the initial motion of the wave packet. (2) for the refractive index near the visible range is fulfilled for the vast majority of materials of interest.

The computational scheme presented above can be used to find the solution of the wave equation with the boundary conditions for $t=0, t=\Delta t, \quad y=0, \quad y=y_{\max }, z=0$, and $z=z_{\max }$ for any suitably discretizable refractive index profile $n(y, z)$. It remains to specify the method of imposing random roughness on the interface. The technique involves defining a real random field $\xi(y)$ as a superposition of some "potentials" $\xi(\mathrm{y})=\Sigma_{\mathrm{j}} \mathrm{v}\left(\mathrm{y}-\mathrm{y}_{\mathrm{j}}\right)$, where each potential is chosen to be a positive half-period of length $L$ of the sine function with peak amplitude $A$ centered on $y_{j}$ and two negative halfperiods of length $L$ each with peak amplitudes $-\mathrm{A} / 2$ centered on $y_{j}-L$ and $y_{j}+L . L$ is the correlation length, and points $y_{j}$ have a uniform distribution on the real line segment $1.5 L \leqslant y \leqslant y_{\max }-1.5 L$. The average of $v(y)$ is zero, and the weighted integral of $v(y)^{2}$ is equal to $\delta^{2}=N(3 L / 4) A^{2}$, where $N$ is the density of random points $y_{j}$ per unit length, and $A$ is the normalization constant. By the Central Limit theorem, as $N \rightarrow \infty$, the probability distribution of $\xi$ tends to the normal (Gaussian) form with variance $\delta^{2}$. The quantity $\delta$ is referred to in this letter as the feature depth. The condition $N \rightarrow \infty$ can be deduced to correspond to $N L \gg 1 .^{8}$ Having obtained $\xi(y)$, we interpret it as the explicit representation of the random surface $z(y)$ in terms of the two adjustable parameters $L$ and $\delta$ (although $\delta$ is a function of $L$, it can be specified independently from it by adjusting $A$ ). It may be further noted at this juncture that the technique outlined here can be applied to other models of mirror roughness as well.

Since the disordered surface specified above is based on a particular pseudorandom sequence of points $y_{j}$ produced by a random number generator, the behavior of the wave packet incident on the surface from the slab waveguide

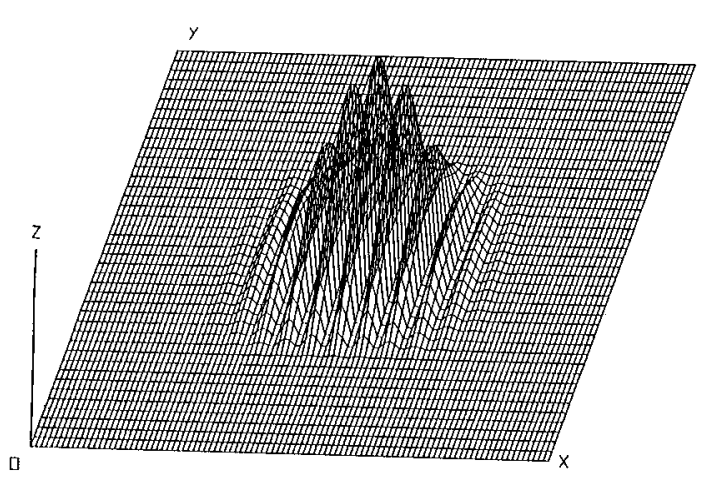

(a)

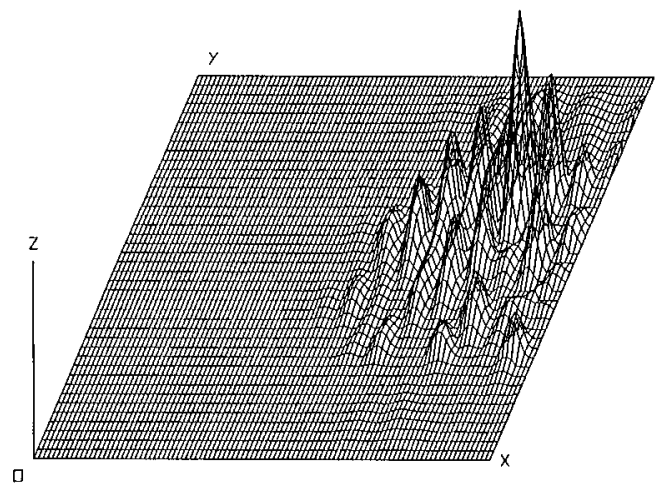

(c)

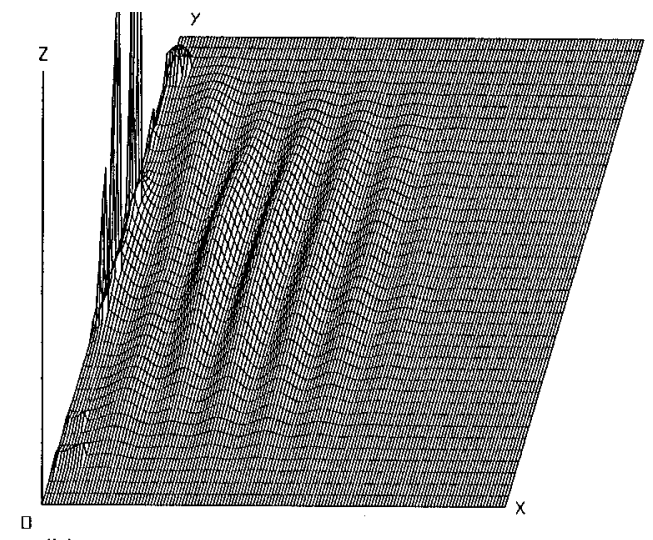

(b)

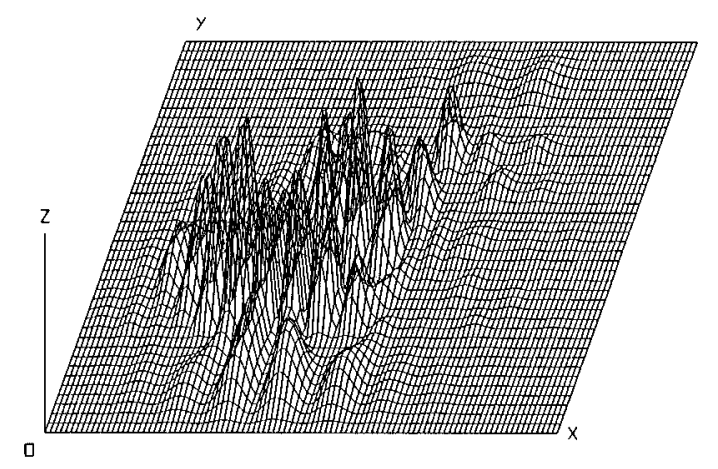

(d)

FIG. 1. The 3D representation of wave packet propagation and reflection from the disordered interface with a correlation length of $0.2 \lambda_{0}$ and a feature depth of $0.3 \lambda_{0}$ : (a) initial wave packet; (b) wave packet transmission into the air region; (c) reflected wave packet near the interface; (d) reflected wave packet away from the interface. 


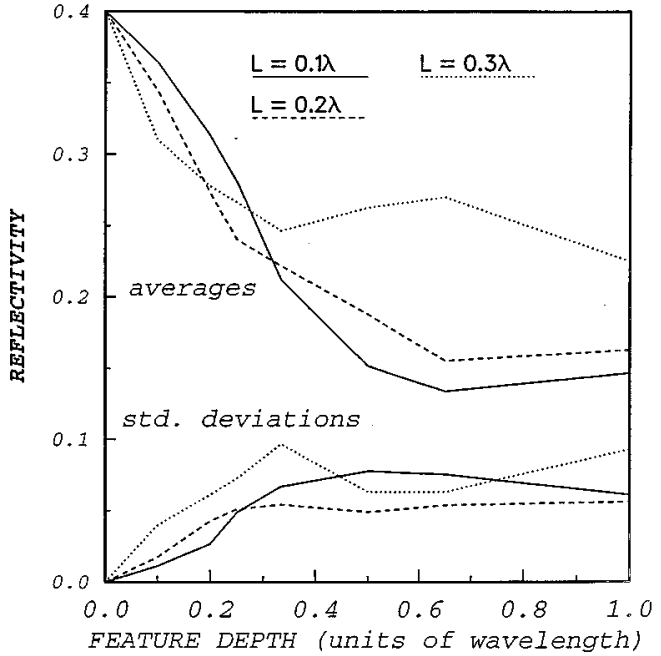

FIG. 2. The averages and standard deviations in the reflectivity for a series of ten simulations.

should be explored in the limit of a large number of repeated simulations with independent pseudorandom number sequences, an approach that falls into the class of Monte Carlo techniques. The waveguide that is selected for the studies presented here is symmetrical with the active layer index of 3.0 and thickness of $\lambda_{0}$. (Since the results are found to scale with the center wavelength provided it remains near the optical range, all lengths are quoted in these units.) The cladding layer refractive index is chosen to be 2.0 for optimum confinement permitting a minimum of mesh points in the transverse direction $\left(\Delta y=0.1 \lambda_{0}\right)$. The form of the initial wave packet is shown in Fig. 1(a). Provided the interface with air $(n=1.0)$ is perfectly smooth, the reflected wave packet preserves the fundamental transverse mode profile apart from a negligible fraction of carriers coupling into other modes. The reflectivity found by $y$ and $z$ integrations is 0.40 , which represents a considerable enhancement over the plane-wave value of 0.25 owing to the strong waveguide confinement. ${ }^{9}$ The transmitted portion of the wave packet diffracts in the air region so that it fills the entire width of the simulated area, while at the time of transmission, large peaks form at the interface [Fig. 1(b)].

Consider now the results of the simulation for a disordered interface. The reflected wave packet [Figs. 1(c) and 1(d)] no longer exhibits the fundamental-mode transverse profile. It is also obvious that some loss of coherence in the longitudinal direction occurs. The value of the reflectivity for this wave packet was evaluated by finding its overlap in both transverse and longitudinal directions with the original wave packet far away from the interface. The convergence of the result has been tested by expanding the boundaries of the simulated region and varying the mesh pitch. The averages and standard deviations from ten runs are shown in Fig. 2 for three values of the correlation length. It is apparent that for a feature depth much less than that, the center wavelength of the reflectivity decreases exponentially with the approximate dependence on the feature depth and the correlation length of the form $\exp \left(-a L \delta / \lambda_{0}^{2}\right)$, where $a \approx 10$. If the feature depth exceeds approximately a third of the center wavelength, the behavior changes qualitatively in that the decrease in the reflectivity levels off at values of the reflectivity increasing with the correlation length. It must be noted, however, that although the results in this range were obtained for the sake of completeness of the study, the state-of-the-art etching technology allows the reduction of interface roughness to several hundred to a thousand microns which is much less than a typical $\lambda_{0}$ of $2800 \AA$ in a material with a refractive index of 3.6. Therefore, the results in the leftmost part of the figure are of much greater practical interest. The standard deviation in that region is found to increase with the feature depth and constitutes anywhere from $5 \%$ to $10 \%$ of the total reflectivity.

Although the results of this study have been presented for a single waveguide configuration, by examining the simulation results from other profiles, we have grounds to believe that the results obtained for a sufficiently small degree of disorder are representative of a wide variety of optical waveguides with rough interfaces. Thus, the major result of our study is that, for disorder on the scale much less than the optical wavelength, the decrease in the reflectivity is only a few percent with the exponential dependence on the feature depth and correlation length. More complex behavior is observed for increased scales of disorder; however, it is primarily of academic interest since currently the etching processes can be controlled quite precisely.

After this letter had been submitted for publication, an experimental study of the lasers with dry-etched mirrors was published, ${ }^{10}$ which indicates that significantly lower thresholds are achievable if the etched mirrors are passivated (see Table II of Ref. 10). Since the passivation step is likely to have much more pronounced effect on the nonradiative recombination at the mirrors than on the mirror roughness, the experimental result appears to support the conclusions of this work.

This work was supported by the ARPA under Contract No. MDA972-94-1-0004.

${ }^{1}$ M. Wada, K. Hamada, T. Shibutani, H. Shimizu, M. Kume, K. Itoh, G. Kano, and I. Teramoto, IEEE J. Quantum Electron. QE-21, 658 (1985).

${ }^{2}$ G. A. Vawter, L. A. Coldren, J. L. Merz, and E. L. Hu, Appl. Phys. Lett. 51, 719 (1987).

${ }^{3}$ J. Puretz, R. K. DeFreez, R. A. Elliott, and J. Orloff, Electron. Lett. 22, 700 (1986).

${ }^{4}$ P. Vettiger, M. K. Benedict, G.-L. Bona, P. Buchmann, E. C. Cahoon, K. Dätwyler, H.-P. Dietrich, A. Moser, H. K. Seitz, O. Voegeli, D. J. Webb, and P. Wolf, IEEE J. Quantum Electron. QE-27, 1319 (1991).

${ }^{5}$ A. Yariv and P. Yeh, Optical Waves in Crystals (Wiley, New York, 1984), Chap. 11.

${ }^{6}$ J. C. Strikwerda, Finite-Difference Schemes and Partial Differential Equations (Wadsworth and Brooks, Pacific Grove, CA, 1989), Chaps. $1-3$.

${ }^{7}$ A. R. Mitchell, Computational Methods in Partial Differential Equations (Wiley, New York, 1969), Chap. 5, Sec. 5.10.

${ }^{8}$ J. M. Ziman, Models of Disorder: The Theoretical Physics of Homogeneously Disordered Systems (Oxford University Press, New York, 1979), Chap. 3.

${ }^{9}$ C. M. Herzinger, C. C. Lu, T. A. DeTemple, and W. C. Chew, IEEE J. Quantum Electron. QE-29, 2273 (1993).

${ }^{10}$ N. C. Frateschi, M. Y. Jow, P. D. Dapkus, and A. F. J. Levi, Appl. Phys. Lett. 65, 1748 (1994). 